\title{
A Novel Theory: Measuring the Real-Time-Based Arterial Properties via Vascular Loading Decoupling Technique
}

\author{
Henry Cherng Han Lin ${ }^{1}$, Albert Chin-Yuh Lin ${ }^{2}$ \\ ${ }^{1}$ School of Chinese Medicine, China Medical University, Taichung, Taiwan \\ ${ }^{2}$ Gong Bu Design Co., Inc., Taichung City, Taiwan \\ Email: albert@fcu.edu.tw
}

How to cite this paper: Lin, H.C.H. and Lin, A.C.-Y. (2018) A Novel Theory: Measuring the Real-Time-Based Arterial Properties via Vascular Loading Decoupling Technique. Health, 10, 1609-1623. https://doi.org/10.4236/health.2018.1011121

Received: September 26, 2018

Accepted: November 27, 2018

Published: November 30, 2018

Copyright (c) 2018 by authors and Scientific Research Publishing Inc. This work is licensed under the Creative Commons Attribution International License (CC BY 4.0).

http://creativecommons.org/licenses/by/4.0/

\begin{abstract}
This paper presents a decoupling theory named as Vascular Loading Decoupling Technique (VLDT), extended from the beat-based Tissue Control Method (TCM), for noninvasive measurement of real-time-based intra-arterial blood pressure and dynamic compliance of blood vessel. Both VLDT and TCM are based upon the decoupling theory to cause the arterial pulsation without the influence from surrounding tissues and measure the variations of the vascular diameter at critical depth. Meanwhile, the AC part of blood pressure is lost, that is, the reference pressure for AC controller is absent as well. To remedy this problem, VLDT employs Step-Hold control rules and cubic spline curve fitting technique to estimate the reference pressure, identify the impedance of blood vessel, and calculate the AC control gain at Hold stage, then track the AC part of blood pressure and compute the real-time arterial blood pressure at Step stage by turns, which makes the real time measurement feasible; moreover, the dynamic compliance of blood vessel can be extracted from real-time impedances of blood vessel. Since the compliance of blood vessel is decoupled from surrounding tissues, it is valuable for diagnosing the severity of vascular sclerosis. The simulation results showed that the VLDT approach is superiority over TCM. This could provide new prospective to circulatory medicine research.
\end{abstract}

\section{Keywords}

Instantaneous Arterial Blood Pressure, Dynamic Compliance of Blood Vessel, Vascular Loading Decoupling Technique, Impedance of Blood Vessel

\section{Introduction}

Since Marey [1] first invented the sphygmograph to measure the blood pressure, 
many non-invasive measuring devices have been developed. Perhaps, Finapres [2] or Portpres is the most popular device in commercial to measure the finger continuous blood pressure. It was originally developed by Peňáz [3] and Wesseling [4] [5] based upon the vascular unloading technique [6] [7] [8]. Later, Yamakoshi [9] [10] [11] [12] [13] proposed the volume-compensation method to improve the servo reference, and developed a local pressurization technique to design a pad-type cuff sphygmomanometer [11] [12] for finger and wrist [13] to avoid the occluding cuff encircling the biological segment that makes it uncomfortable in long-term measurements.

Based upon the model for transmission of arterial blood pressure [14], none of present non-invasive measuring devices is able to measure the actual instantaneous blood pressure in an artery, mainly because these methods are not capable of determining the transmission characteristics of the tissue and blood vessel. A possible approach to obtain these characteristics is to decouple the pulsation of the blood vessel from the tissues by using control and identification techniques.

Therefore, Lin [14] developed an innovative blood pressure measurement technique, named Tissue Control Method (TCM), which is by maintaining the DC part of blood pressure and tracking the AC part of blood pressure to cause the vascular truly unloading; the blood vessel is pulsated without the influence of surrounding tissues. Accordingly, the variation of blood vessel diameter is obtained, but meanwhile, the AC part of blood pressure is lost, that is, the reference pressure for controller is absent as well. For estimating the impedance of blood vessel, the self-adaptive control algorithm is adopted, and the peak-to-peak blood pressure of the previous pulse is taken as reference pressure, by which, the beat-based intra-arterial blood pressure could be obtained, but obviously the value is inaccurate; in addition, the obtained impedance of blood vessel is just an approximate mean value in one pulse pressure cycle. In other words, although TCM method could lead to the unloading state of the blood vessel, the absence of reference pressure causes the intra-arterial blood pressure and the dynamic compliance of the blood vessel to be unobtainable.

To remedy this problem, we proposed a new approach named as real-time based Vascular Unloading Decoupling Technique (VLDT). It employs the Step-Hold control rules and featured spline curve fitting technique to estimate the reference pressure and measure the impedance of blood vessel. It makes the measurements of real time intra-arterial blood pressure and the dynamic compliance of blood vessel feasible.

\section{Models and Theory}

\subsection{Mechanical Circuit Model for Transmission of Arterial Blood Pressure}

The models and theory of the Tissue Control Method is restated in this paragraph. Figure 1 describes the physical model of the arterial blood pressure at the radial artery. The point at detection head is labeled $n$, the skin surface is labeled 


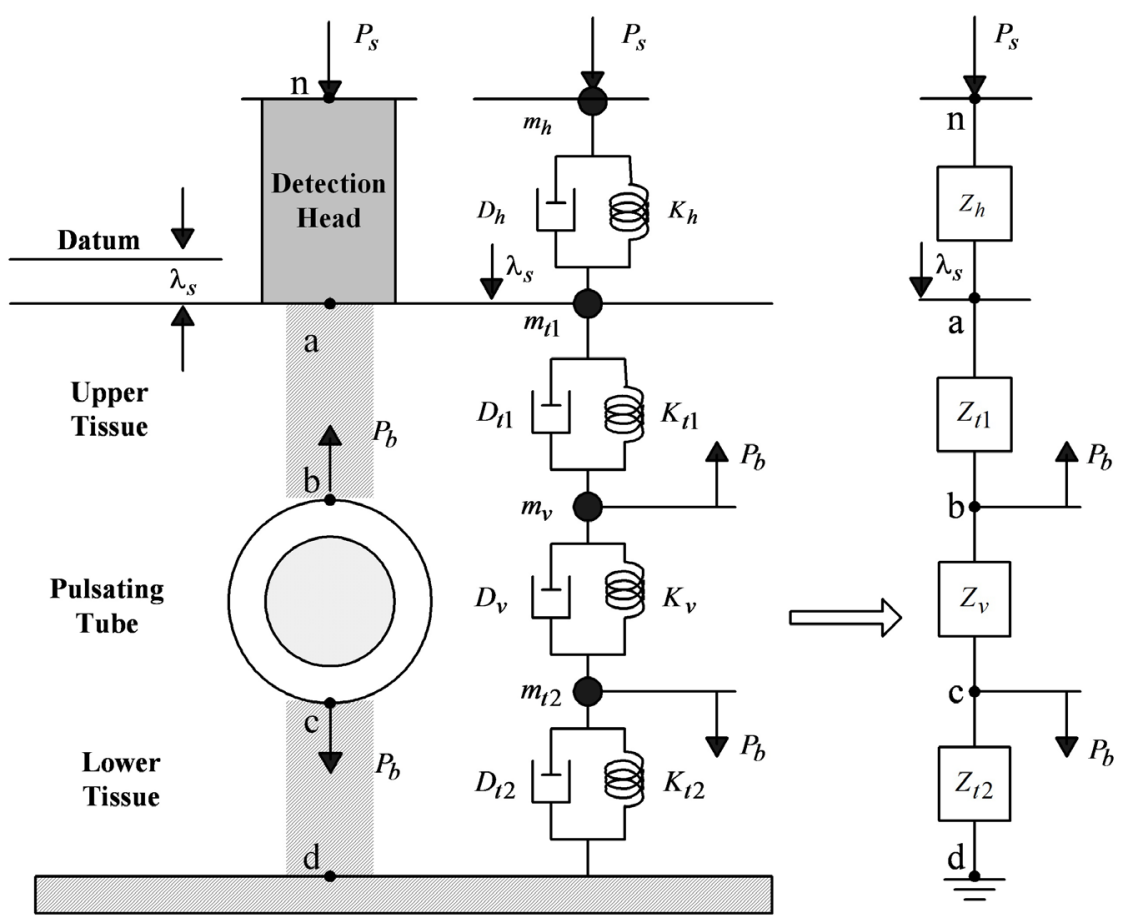

Figure 1. The lumped mechanical circuit model for transmission of arterial blood pressure.

a, the surface of the radius is $d$ and the points $b$ and $c$ are the blood vessel's upper and lower points respectively. Assuming that the motion model of the arterial blood pressure is lumped, one may express each part, from the detection head to the radius, by a mass element $(M)$, a damping element $(D)$ and a stiffness element $(K)$ to describe the motion behavior caused by arterial blood pressure transmitted from inside the vessel to the skin above. If the force transmissibility is equivalent to the pressure transmissibility from the blood vessel to the skin, the elements of $M, D$ and $K$ may be defined as

$$
M=\frac{P_{M}}{\ddot{\lambda}}, \quad D=\frac{P_{D}}{\dot{\lambda}}, \quad K=\frac{P_{K}}{\lambda}
$$

where $P$ is the pressure and $\lambda$ is the displacement. Then, by using the Laplace transform, one may define the impedance $Z$ for each part as

$$
Z(s)=\frac{P}{\lambda}=M s^{2}+D s+K
$$

If the subscripts $h, t 1, v$ and $t 2$ denote the parts of detection head, upper tissue, blood vessel and lower tissue, respectively, then the symbols of $Z_{h}, Z_{t 1}, Z_{v}$ and $Z_{t 2}$ indicate the impedances for the corresponding parts of the lumped model.

If the displacement $\lambda$ is analogous to the voltage and the pressure $P$ is analogous to the current, then the mechanical impedance will be analogous to the electrical admittance.

Figure 2(a) shows the equivalent mechanical circuit model for the transmission of arterial blood pressure at the radial artery. One may further combine the 


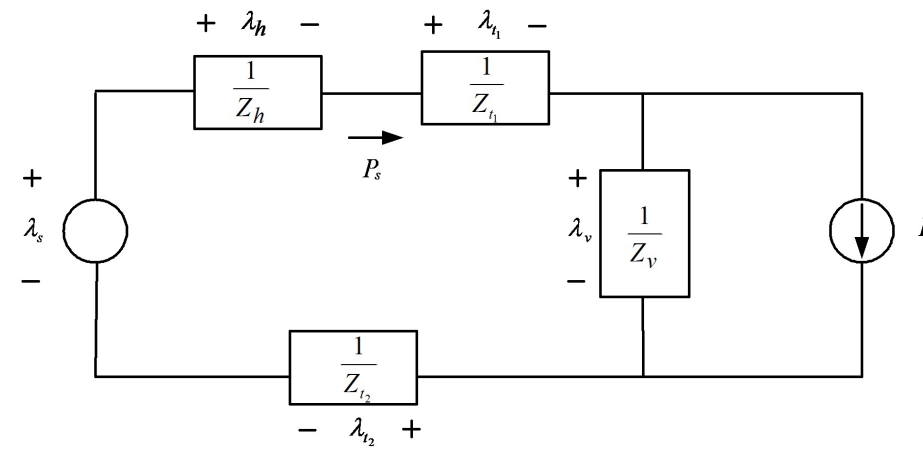

(a)

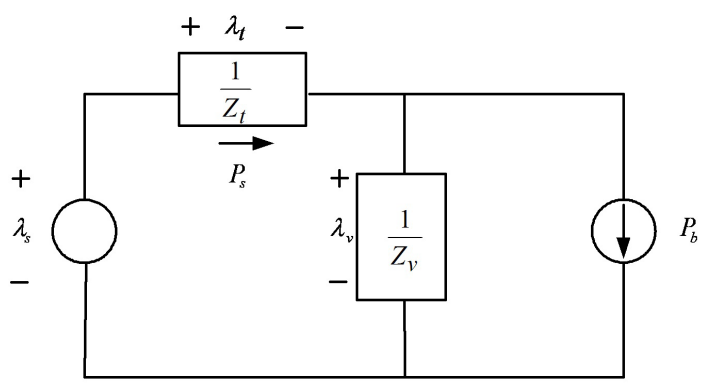

(b)

Figure 2. The mechanical circuit model for the transmission of arterial blood pressure under pressed conditions.

impedances of the detection head $\left(Z_{h}\right)$, upper tissue $\left(Z_{t 1}\right)$ and the lower tissue $\left(Z_{t 2}\right)$ as the impedance of tissue $\left(Z_{t}\right)$ to simplify the circuit, as shown in Figure 2(b). However, it should be noted that the experimental data show that the impedances are not constants as they vary with the pressed depth.

$$
\frac{1}{Z_{t}}=\frac{1}{Z_{h}}+\frac{1}{Z_{t 1}}+\frac{1}{Z_{t 2}}
$$

The abovementioned detection head can be a cuff or an artery tonometry with actuators and sensors.

\subsection{Analysis of Pulsation of Tissue and Artery}

According to the superposition theorem, one may express the response of the blood pressure measured on the skin $\left(P_{s}\right)$ due to excitations of the pressed-down depth $\left(\lambda_{s}\right)$ and the arterial blood pressure $\left(P_{b}\right)$ as

$$
P_{s}=\frac{Z_{t} Z_{v}}{Z_{t}+Z_{v}} \lambda_{s}+\frac{Z_{t}}{Z_{t}+Z_{v}} P_{b}
$$

where the arterial blood pressure $P_{b}$ can be summed by DC part of blood pressure $\left(\bar{P}_{b}\right)$ and the AC part of blood pressure $\left(\Delta P_{b}\right)$.

\subsubsection{Analysis of DC Part of Blood Pressure Excitation ( $\bar{P}_{b}$ )}

If one presses the skin to a critical depth $\left(\bar{\lambda}_{s}\right)$ such that the DC part of sensor's pressure $\left(\bar{P}_{s}\right)$ measured on the skin equals the DC part of arterial blood pressure $\left(\bar{P}_{b}\right)$, then (4) can be written as

$$
\bar{P}_{s}=\frac{\bar{Z}_{t} \bar{Z}_{v}}{\bar{Z}_{t}+\bar{Z}_{v}} \bar{\lambda}_{s}+\frac{\bar{Z}_{t}}{\bar{Z}_{t}+\bar{Z}_{v}} \bar{P}_{b}, \quad \bar{P}_{s}=\bar{P}_{b}
$$

Solving (5) gives

$$
\bar{P}_{s}=\bar{Z}_{t} \bar{\lambda}_{s}=\bar{P}_{b} \quad \Rightarrow \bar{Z}_{t}=\frac{\bar{P}_{s}}{\bar{\lambda}_{s}}=\bar{Z}_{s}
$$

Obviously, (6) states that at the critical depth $\left(\bar{\lambda}_{s}\right)$ where, the impedance measured on the skin is equal to the equivalent impedance of detection head, upper tissue and lower tissue only, and the impedance of blood vessel is no 
longer involved in the analysis. It further illustrates that the impedance of arterial blood vessel $\left(\bar{Z}_{v}\right)$ and the impedance of other surrounding tissues $\left(\bar{Z}_{t}\right)$ are decoupled.

\subsubsection{Analysis of AC Part of Blood Pressure Excitation $\left(\Delta P_{b}\right)$}

When the AC part of arterial blood pressure $\left(\Delta P_{b}\right)$ is imposed on the DC part of the arterial blood pressure $\left(\bar{P}_{b}\right)$ at critical depth $\left(\bar{\lambda}_{s}\right),(4)$ can be rewritten as

$$
\bar{P}_{s}+\Delta P_{s}=\frac{Z_{t} Z_{v}}{Z_{t}+Z_{v}}\left(\bar{\lambda}_{s}+\Delta \lambda_{s}\right)+\frac{Z_{t}}{Z_{t}+Z_{v}}\left(\bar{P}_{b}+\Delta P_{b}\right)
$$

Substituting (6) into (7) yields

$$
\Delta P_{s}=\frac{Z_{t} Z_{v}}{Z_{t}+Z_{v}}\left(1-\frac{\bar{Z}_{t}}{Z_{t}}\right) \bar{\lambda}_{s}+\frac{Z_{t} Z_{v}}{Z_{t}+Z_{v}} \Delta \lambda_{s}+\frac{Z_{t}}{Z_{t}+Z_{v}} \Delta P_{b}
$$

If one moves the detection head up and down $\left(\Delta \lambda_{s}\right)$ at critical depth $\left(\bar{\lambda}_{s}\right)$ to keep the AC part of the sensor's pressure measured on the skin $\left(\Delta \bar{P}_{s}\right)$ is equal to zero, i.e. $\Delta \bar{P}_{s}=0$ and then the impedance of surrounding tissues remains unchanged, i.e. $Z_{t}=\bar{Z}_{t}$, because it compressed by $\bar{P}_{s}=\bar{P}_{b}$ only. Then (8) becomes

$$
\Delta \lambda_{s}=-\frac{1}{Z_{v}} \Delta P_{b}=\Delta \lambda_{v} \quad \text { or } \quad \Delta P_{b}=-Z_{v} \Delta \lambda_{s}
$$

where $\Delta \lambda_{v}$ is the variation of the diameter of blood vessel. It also represents that the movements of blood vessel under this situation is varying with zero transmural pressure, because the artery's inside and outside pressures are equal, $\bar{P}_{s}=\bar{P}_{b}$, that is truly vascular unloading. This derivation reveals that the variation of the displacement measured on the skin $\left(\Delta \lambda_{s}\right)$ is equivalent to the variation of the diameter of blood vessel $\left(\Delta \lambda_{v}\right)$ due to tracking AC part of the arterial blood pressure, but meanwhile, the AC part of blood pressure is lost, $\Delta P_{b} \approx 0$.

However, the significant implication of this result is that the pulsation of blood vessel is therefore decoupled from the surrounding tissues which enable one may identify the impedance of blood vessel $\left(Z_{v}\right)$ and then compute the intra-arterial blood pressure as shown in (10).

$$
P_{b}=\bar{P}_{b}+\Delta P_{b}=\bar{P}_{s}-Z_{v} \Delta \lambda_{s}
$$

For obtaining the situation of (10), we present the real-time-based Vascular Loading Decoupling Technique (VLDT) instead of the beat-based Tissue Control Method (TCM) to detect the dynamic impedance of blood vessel and the instantaneous intra-arterial blood pressure.

\section{Vascular Loading Decoupling Technique}

Three key techniques are used for the VLDT. There are 1) separating the arterial blood pressure into two parts: DC part of blood pressure $\left(\bar{P}_{b}\right)$ and the AC part of blood pressure $\left(\Delta P_{b}\right)$ and using self-adaptive control rules to create the decoupled situation to provide the possibility of measuring the impedances of surrounding tissues and blood vessel respectively; 2) using Step-Hold control rules to 
estimate reference pressure, identify the impedance of blood vessel, track the AC part of blood pressure and compute the real-time intra-arterial blood pressure; 3 ) extracting the dynamic compliance of blood vessel $\left(C_{3}\right)$ from the impedance of blood vessel $\left(Z_{V}\right)$ by using parameter estimation technique.

Figure 3 is a control diagram for VLDT, where the parallel impedance $\left(H_{1}\right)$ and the dividend impedance $\left(H_{2}\right)$ is the plant, and $G_{d}$ is the block diagram of DC controller and DC-driven actuator and $G_{a}$ is the $\mathrm{AC}$ controller and the AC-driven actuator respectively. Two displacement sensors, DC part of displacement sensor and AC part of displacement sensor are attached with DC-driven actuator and AC-driven actuator and sensing their movements $\bar{\lambda}_{s}$ and $\Delta \lambda_{v}$ respectively. Whereas, the pressure $P_{s}$ measured by the pressure sensor is processed by a FUNC program to obtain a DC part of sensor's pressure $\left(\bar{P}_{s s}\right)$ and an AC part of sensor's pressure $\left(\Delta P_{s s}\right)$, which are taken as feedback signals for DC controller and $\mathrm{AC}$ controller, respectively.

\subsection{Creating the Decoupling Situation by Controlling the Arterial Blood Pressure}

Observing Figure 3, the response of $P_{s}$ is the result of the excitations of the DC part and the AC part of arterial blood pressure. To create a decoupling situation, we need performing DC control to maintain the arterial pulsation at critical depth and tracking the AC part of blood pressure via AC control. Therefore, the system response can be analyzed individually.

\subsubsection{System Response Due to the Excitation of DC Part of Blood Pressure}

Figure 4 is a block diagram for DC control of VLDT. The purpose of DC control is to maintain the detection head at critical depth $\left(\bar{\lambda}_{s}\right)$ where the DC part of sensor's pressure is equal to the DC part of arterial blood pressure, $\bar{P}_{s s}=\bar{P}_{b}$. In order to limit the measuring error of DC part of arterial blood pressure and the nonlinear of impedances of surrounding and blood vessel, we proposed the self-adaptive control rule to set the DC open loop gain $\left(K_{d}\right)$ as constant, where $K_{d}=G_{d} H_{1}$. Based upon control theory, the DC part of sensor's pressure $\left(\bar{P}_{s s}\right)$ can be expressed as (11).

$$
\bar{P}_{s s}=\frac{G_{d} H_{1}+H_{2}}{1+G_{d} H_{1}} \bar{P}_{b}
$$

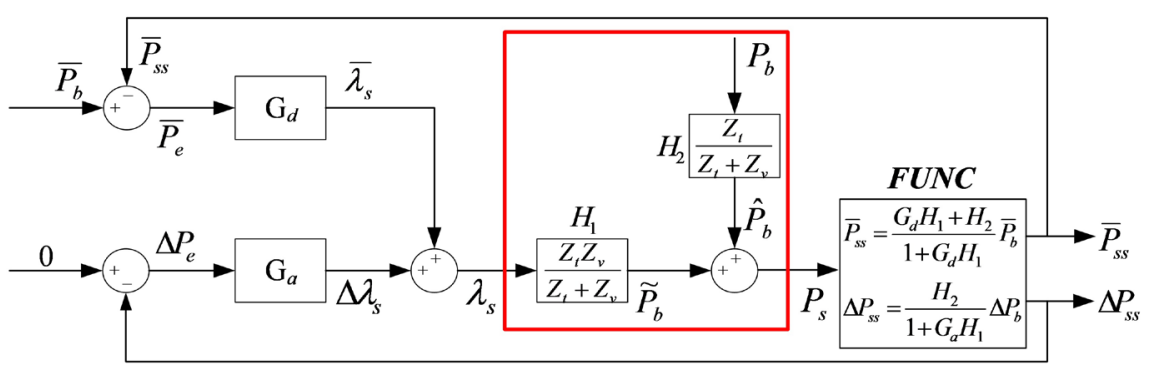

Figure 3. The control diagram for Vascular Loading Decoupling Technique. 


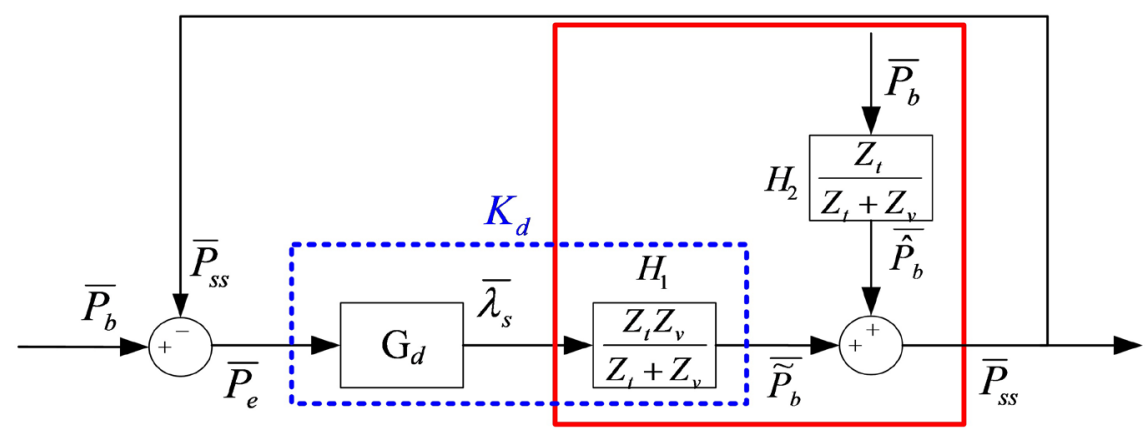

Figure 4. The control diagram for maintaining DC part of arterial blood pressure.

where the dividend impedance $H_{2}=Z_{t} /\left(Z_{t}+Z_{v}\right)$ is smaller than 1 .

Therefore, set the DC open loop gain $K_{d}=G_{d} H_{1}=499$, the error of DC control results in (11) is less than $0.2 \%$. It means that setting the DC control gain $\left(G_{d}\right)$ to have DC open loop gain $\left(K_{d}\right)$ is constant will keep the detection head nearly to the critical depth, which is similar to the analysis of DC part of arterial blood pressure excitation.

\subsubsection{System Response Due to the Excitation of AC Part of Blood Pressure}

When the DC-driven actuator is pressed to reach the critical depth $\left(\bar{\lambda}_{s}\right)$, an AC controller is needed to move up and down the AC-driven actuator with displacement $\left(\Delta \lambda_{s}\right)$ in order to have the AC part of sensor's pressure be zero, $\Delta P_{s s}=0$, as shown in Figure 5.

The control theory gives the AC part of sensor's pressure $\left(\Delta P_{s s}\right)$ shown in (12).

$$
\Delta P_{s s}=\frac{H_{2} \Delta P_{b}}{1+G_{a} H_{1}}
$$

Similarly, the self-adaptive control rule is used. Adjust the AC control gain $\left(G_{a}\right)$ so as to maintain the AC open loop gain to be the fixed value, $G_{a} H_{1}=K_{a}$. If set $K_{a}=199$, AC part of sensor's pressure $\left(\Delta P_{s s}\right)$ is smaller than $0.5 \%$, i.e. $\Delta P_{s s} \approx 0$. By now, the pulsation of arterial blood vessel at critical depth is similar to the analysis of AC part of arterial blood pressure $\left(\Delta P_{b}\right)$.

\subsection{Using Step-Hold Control Rules to Identify the Impedance of Blood Vessel and Compute the Intra-Arterial Blood Pressure}

From Figure 6, it learns that the relations between the reference pressure $\left(\Delta \hat{P}_{b}\right)$ and the parallel impedance $\left(H_{1}\right)$ can be expressed as follows:

$$
\Delta \hat{P}_{b}(n)=\Delta P_{s s}(n)-\Delta \tilde{P}_{b}(n)=-\Delta P(n)-\Delta P_{e}(n-1) G_{a}(n-1) H_{1}(n)
$$

Then the parallel impedance $\left(H_{1}\right)$ can be measured by (13).

$$
H_{1}(n)=\frac{-\Delta P_{e}(n)-\Delta \hat{P}_{b}(n)}{\Delta P_{e}(n-1) G_{a}(n-1)}
$$

However, Figure 6 states that the AC part of sensor's pressure $\left(\Delta P_{s s}\right)$ is the 


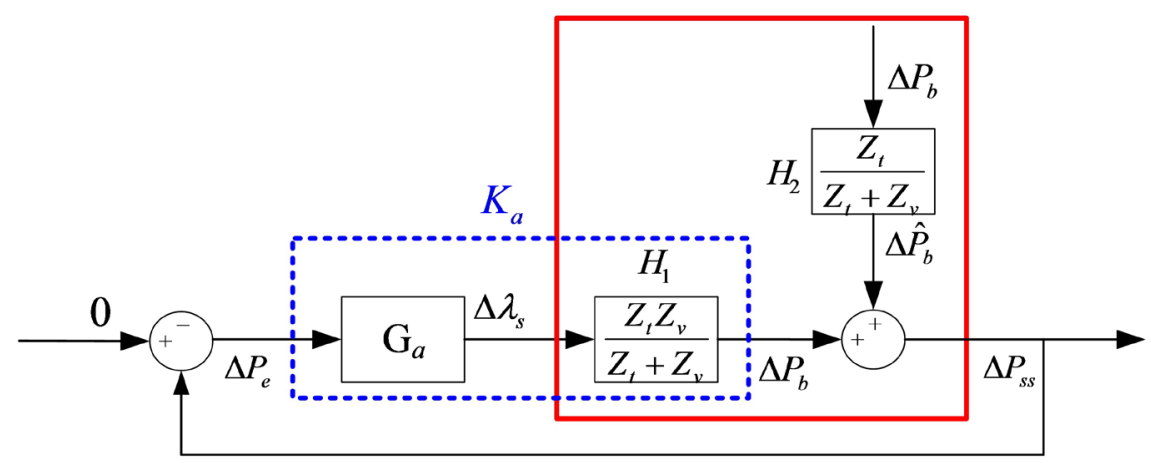

Figure 5. The control diagram for tracking AC part of blood pressure.

(1)

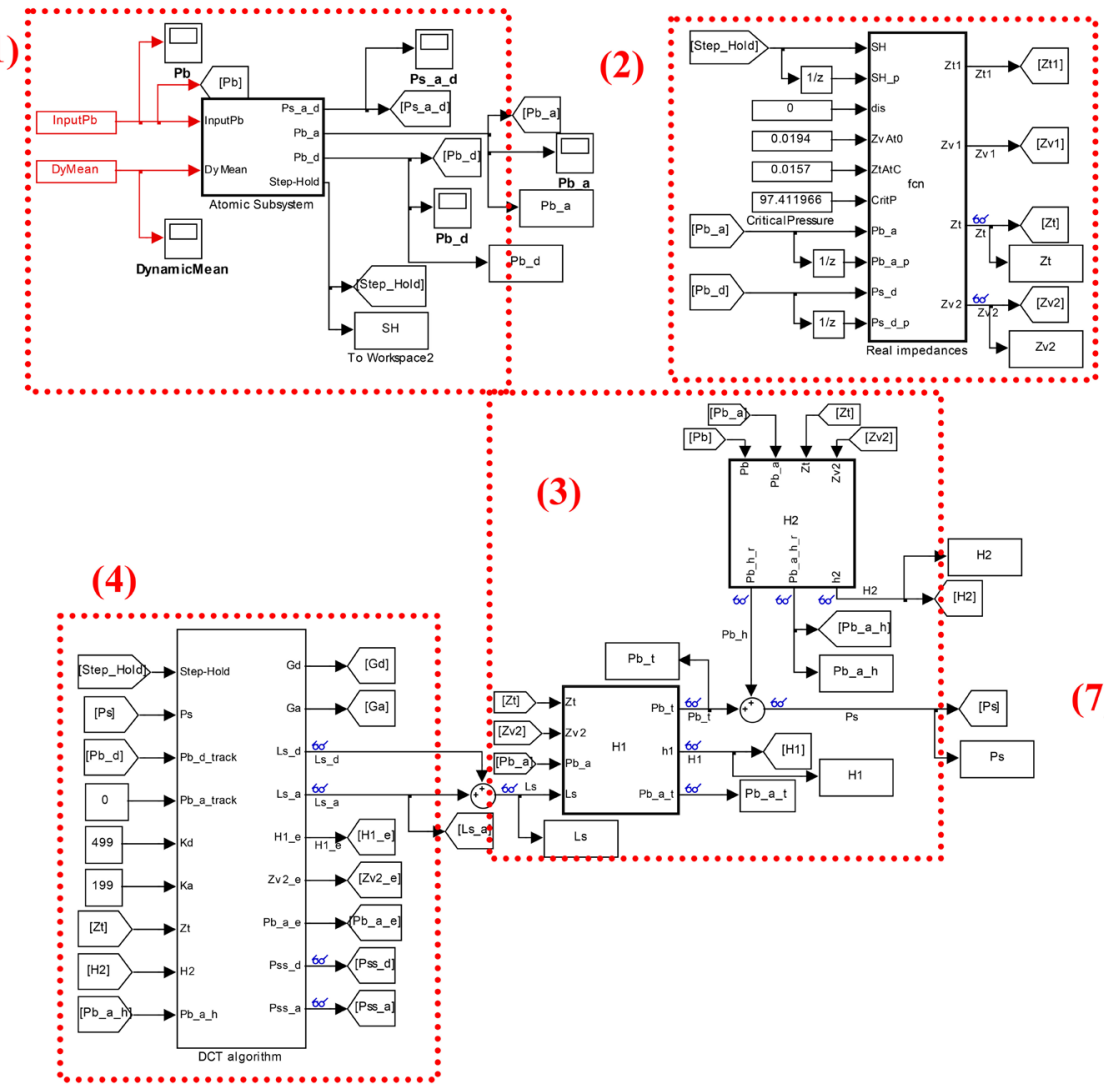

(5)

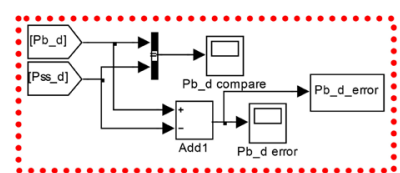

(6)

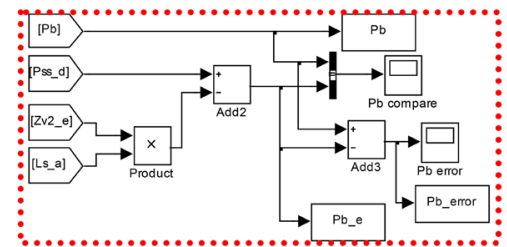

(7)

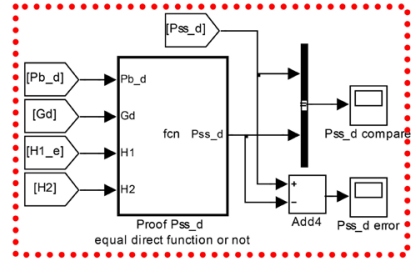

Figure 6. The framework for VLDT simulation block diagram by Simulink software.

sum of the reference pressure $\left(\Delta \hat{P}_{b}\right)$ and the output pressure $\left(\Delta \tilde{P}_{b}\right)$ of parallel impedance $\left(H_{1}\right), \Delta P_{s s}=-\Delta P_{e}=\Delta \hat{P}_{b}+\Delta \tilde{P}_{b}$. The reference pressure $\left(\Delta \hat{P}_{b}\right)$ cannot be measured unless the output pressure $\left(\Delta \tilde{P}_{b}\right)$ of parallel impedance $\left(H_{1}\right)$ to be zero. Therefore, we provided Step-Hold control rules to cope with this problem. The period of either Step or Hold stage lasts 2 millisecond, which is at the frequency rate of $500 \mathrm{~Hz}$. At Hold stage, let AC-driven actuator is idling, the AC part of displacement $\left(\Delta \lambda_{s}\right)$ is zero and causes the output pressure $\left(\Delta \tilde{P}_{b}(n-1)\right)$ of parallel impedance is zero, then the measurement of AC part of sensor's pressure is equal to the reference pressure, $\Delta P_{s s}(n-1)=\Delta \hat{P}_{b}(n-1)$. 
Use three Hold stages at beginning to have three instant reference pressures $\Delta \hat{P}_{b}(n-3), \Delta \hat{P}_{b}(n-2)$, and $\Delta \hat{P}_{b}(n-1)$, and employ the featured cubic spline curve fitting technique to estimate the nth stage of reference pressure $\left(\Delta \hat{P}_{b}(n)\right)$, then compute the impedance of blood vessel $\left(Z_{v}(n)\right)$ at Hold stage by (13) and (14).

$$
\frac{1}{Z_{v}(n)}=\frac{1}{H_{1}(n)}-\frac{1}{Z_{t}(n)}
$$

and the AC control gain $\left(G_{a}(n)\right)$ is obtained by (15) as well.

$$
G_{a}(n)=\frac{K_{a}}{H_{1}(n)}
$$

After that, go to Step stage ( $\mathrm{n}^{\text {th }}$ stage) to actuate the AC-driven actuator with AC control gain $\left(G_{a}(n)\right)$ that maintains the AC part of sensor's pressure is equal to the one of $\left(1+K_{a}\right)$ of reference pressure, i.e.

$\Delta P_{s s}(n)=-\Delta \hat{P}_{b}(n) /\left(1+K_{a}\right)$ and earn variation of blood vessel diameter from AC part of displacement sensor, i.e. $\Delta \lambda_{s}(n) \cong \Delta \lambda_{v}(n)$.

Finally, (16) gives the $\mathrm{n}^{\text {th }}$ stage of intra-arterial blood pressure.

$$
P_{b}(n)=\bar{P}_{s}(n)-\Delta \lambda_{s}(n) Z_{v}(n)
$$

Repeat the Hold and Step stages to obtain the impedance of blood vessel $\left(Z_{v}(n)\right)$ and the arterial blood pressure $\left(P_{b}(n)\right)$ at each cycle until to the end of the measurement.

\subsection{Extracting the Dynamic Compliance of Blood Vessel Data $\left(C_{3}\right)$ from the Impedance of Blood Vessel $\left(Z_{v}\right)$}

In the light of the definition of impedance $Z_{\nu}$, the equivalent mechanical lumped elements, such as mass $\left(M_{v}\right)$, damping $\left(D_{v}\right)$ and stiffness $\left(K_{v}\right)$ constitute (17) as follows:

$$
-Z_{v}(s) \Delta \lambda_{s}^{\prime}=\Delta P_{b}^{\prime} \Rightarrow-\left(M_{v} s^{2}+D_{v} s+K_{v}\right) \Delta \lambda_{s}^{\prime}=\Delta P_{b}^{\prime}
$$

Through bilinear transform, matrix parameters of mass $\left(M_{v}\right)$, damping $\left(D_{v}\right)$ and stiffness $\left(K_{v}\right)$ can be identified and shown in (18):

$$
\left[\begin{array}{c}
M \\
D \\
K
\end{array}\right]=\left[\begin{array}{ccc}
\Delta \lambda_{s}(n) & \Delta \lambda_{s}(n-1) & \Delta \lambda_{s}(n-2) \\
\Delta \lambda_{s}(n-1) & \Delta \lambda_{s}(n-2) & \Delta \lambda_{s}(n-3) \\
\Delta \lambda_{s}(n-2) & \Delta \lambda_{s}(n-3) & \Delta \lambda_{s}(n-4)
\end{array}\right]^{-1}\left[\begin{array}{c}
Z_{v}(n)+2 Z_{v}(n-1)+Z_{v}(n-2) \\
Z_{v}(n-1)+2 Z_{v}(n-2)+Z_{v}(n-3) \\
Z_{v}(n-2)+2 Z_{v}(n-3)+Z_{v}(n-4)
\end{array}\right]
$$

The mass $\left(M_{v}\right)$, damping $\left(D_{v}\right)$ and stiffness $\left(K_{v}\right)$ of blood vessel can be further extracted by (19) as follows:

$$
M_{v}=\frac{T^{2}}{16}(M-D+K) ; \quad D_{v}=\frac{T}{4}(M-K) ; \quad K_{v}=\frac{1}{4}(M+D+K)
$$

where $T$ is the sampling period.

The reciprocal of the stiffness $\left(K_{v}\right)$ is the dynamic compliance of blood vessel, which is defined as $C_{3}$. It is different from the Artery Elasticity Indexes of $C_{1}$ and $C_{2}$ provided by HDI CvProfilor [15], calculated via modified Windkessel Model 
based upon the continuous arterial blood pressure. It means that $C_{3}$ is directly obtained from the AC part of blood pressure and the variation of blood vessel diameter that meets the definition of compliance. Besides, it is measured under the solely pulsation of the arterial blood vessel without the influence of surrounding tissues. It can truly reflect the compliance of blood vessel. Therefore, $C_{3}$ is more reliable for diagnosing the severity of vascular sclerosis.

\section{Simulation and Validation}

To verify the feasibility of VLDT and the superiority over TCM, the framework of the simulation program run by Simulink software shown in Figure 6. It contains seven sections: 1) pressure input section, 2) impedance calculation section, 3) control plant section, 4) VLDT algorithm section, 5) DC part of arterial blood pressure verification section, 6) arterial blood pressure verification section and 7) control formula verification section.

Thirty-five previous TCM experimental data sets give each set of arterial blood pressure are used as the real intra-arterial blood pressures and each set of mean impedances of surrounding tissues and blood vessel are used and assumed as parabolic-like curve for simulation.

\subsection{Estimating the Reference Pressure by Featured Spline Curve Fitting Technique}

VLDT proposed the featured cubic spline curve fitting technique to estimate the reference pressure. It segments the estimated spline curves as a combination of monotonic functions by featured points. The featured points are selected as the points where the function value is zero or the nth derivative of function equals to zero, $\mathrm{n}=1,2$, and 3 . This effectively avoids the occurrence of data scattering or abnormal peaks and robust for estimation. A typical simulation result is shown in Figure 7. The maximum error of thirty-five estimated data sets for reference pressure $\Delta \hat{P}_{b}$ is $0.1689 \mathrm{mmHg}(0.6775 \%)$ and the mean error is $0.0021 \mathrm{mmHg}$ $(0.0635 \%)$.

\subsection{Computing Impedance of Blood Vessel and Intra-Arterial Blood Pressure}

Once the estimated reference pressure is accurate enough, then the results of the impedance of blood vessel and the intra-arterial blood pressure will be satisfied as well. Figure 8 and Figure 9 show the typical simulation results for impedance of blood vessel $Z_{V}$ and intra-arterial blood pressure $P_{b}$. The estimation error of 35 data sets for the impedance of blood vessel $Z_{V}$ is almost zero, and the maximum error for estimated intra-arterial blood pressure $P_{b}$ is $0.2276 \mathrm{mmHg}$ (1.097\%) and the mean error is $0.0863 \mathrm{mmHg}(0.0786 \%)$.

\subsection{Extracting $M_{v}, D_{v}$ and $K_{v}$ from the Impedance of Blood Vessel}

According to (18) and (19), the lumped parameters of mass $\left(M_{v}\right)$, damping $\left(D_{v}\right)$ 
and stiffness $\left(K_{v}\right)$ are extracted as shown in Figure 10. The average values for $M_{v}$, $D_{V}$ and $K_{V}$ are calculated as $2 \times 10^{-9} \mathrm{mmHg} / \mu \mathrm{m} / \mathrm{sec}^{2}, 6 \times 10^{-5} \mathrm{mmHg} / \mu \mathrm{m} / \mathrm{sec}$ and $0.02 \mathrm{mmHg} / \mu \mathrm{m}$ respectively. Then the mean compliance of blood vessel $\left(C_{3}\right)$ is 50 $\mu \mathrm{m} / \mathrm{mmHg}$ which can be used for diagnosing the severity of vascular sclerosis.

\subsection{Comparison the Simulation Results between VLDT and TCM}

In order to reveal the superiority of VLDT over TCM, Figure 11 and Figure 12 are the results simulated by using the beat-based TCM. The estimated error of intra-arterial blood pressure is about $\pm 2 \mathrm{mmHg}$. Comparing with Figure 8 and Figure 9 respectively, it obviously indicates that the real-time-based VLDT is not only more accurate, but also provide a dynamic impedance of blood vessel not the average one at each beat of pulsations by TCM.

To summarize the superiority of VLDT over TCM, they are shown in Table 1.

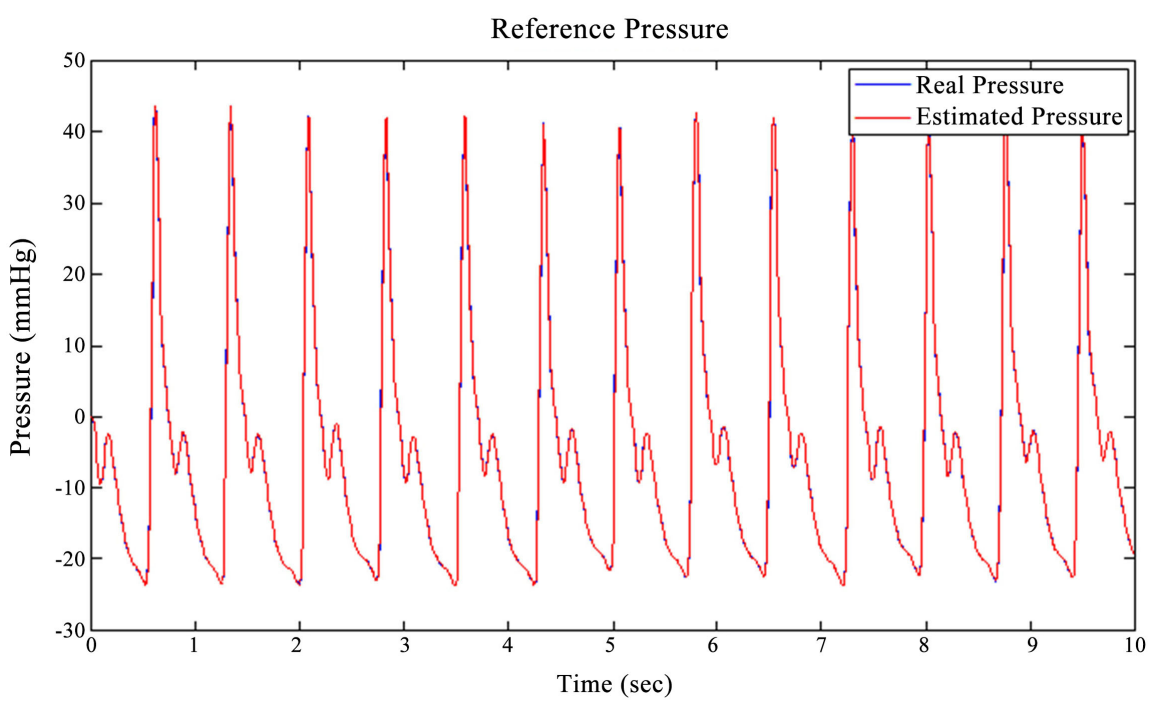

Figure 7. The comparison chart for reference pressure (blue line) and estimated reference pressure (red line).

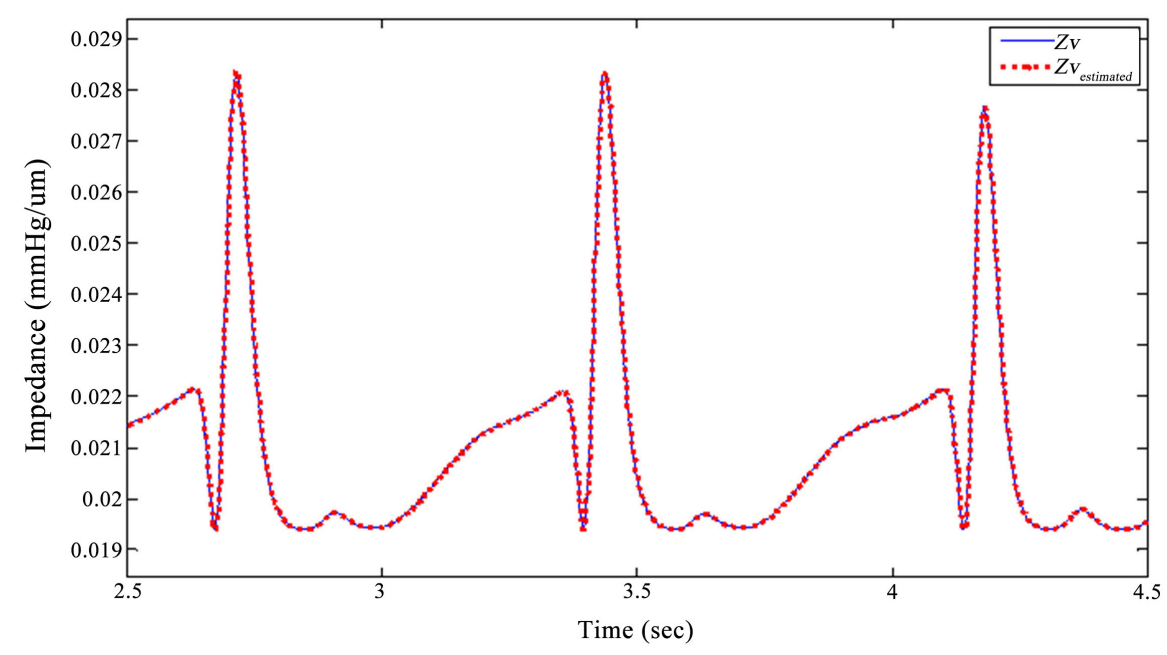

Figure 8. The comparison chart for real and estimated impedance of blood vessel. 


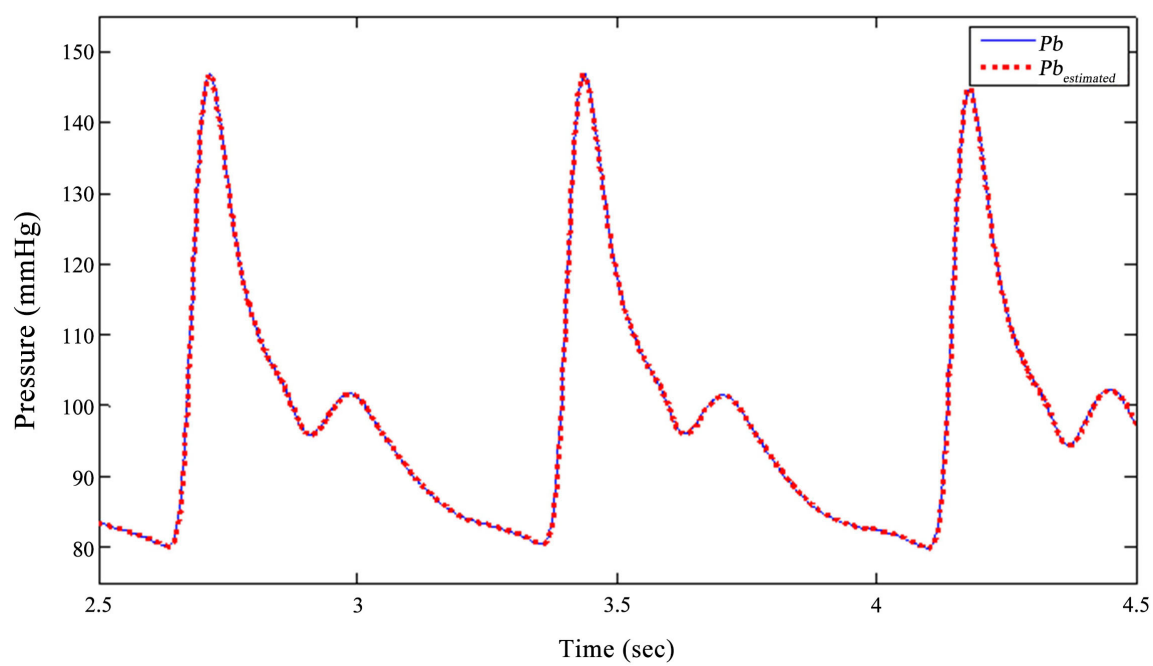

Figure 9. The comparison chart for real and estimated intra-arterial blood pressure.
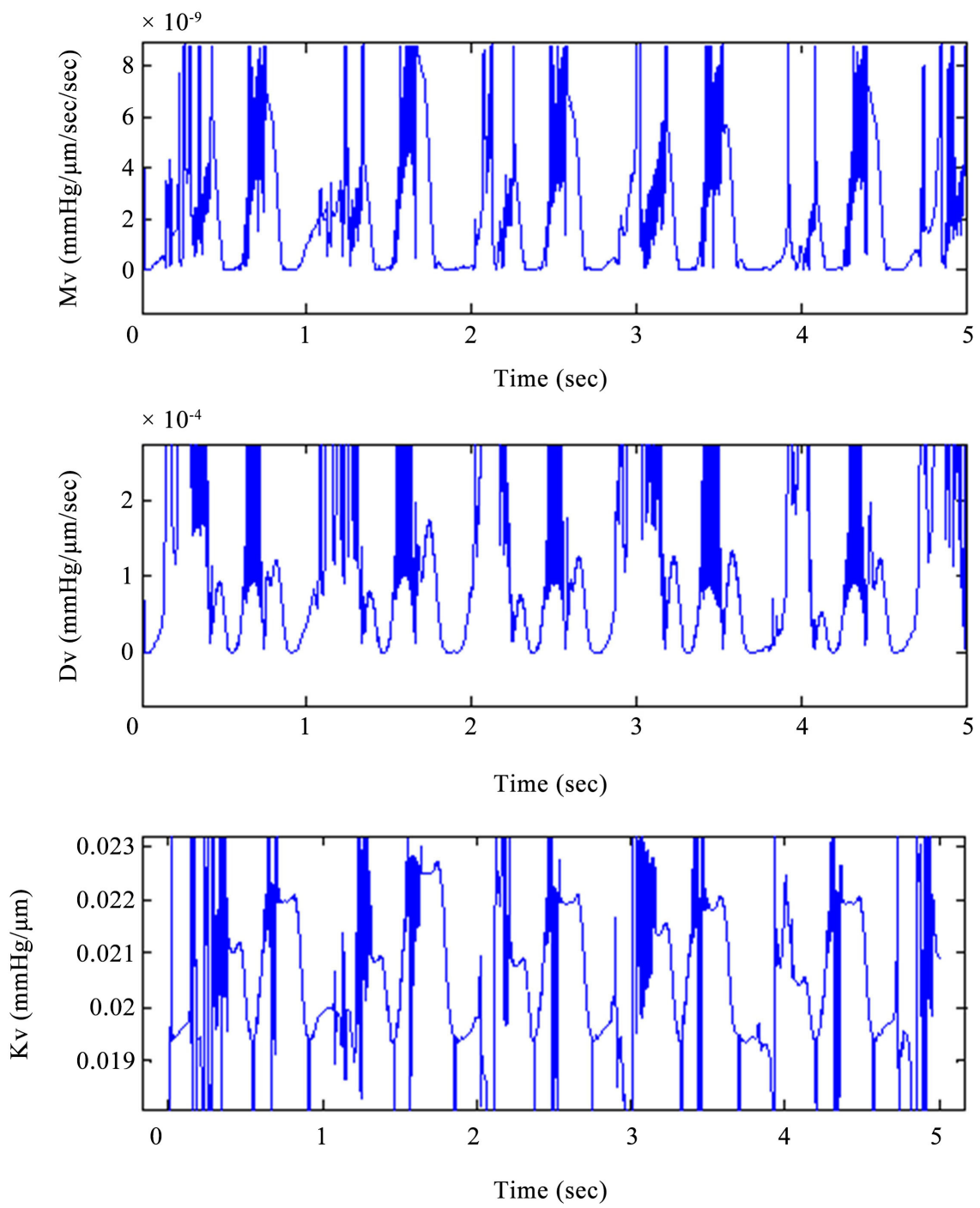

Figure 10. The lumped parameters estimation of mass $\left(M_{v}\right)$, damping $\left(D_{v}\right)$ and stiffness $\left(K_{v}\right)$. 


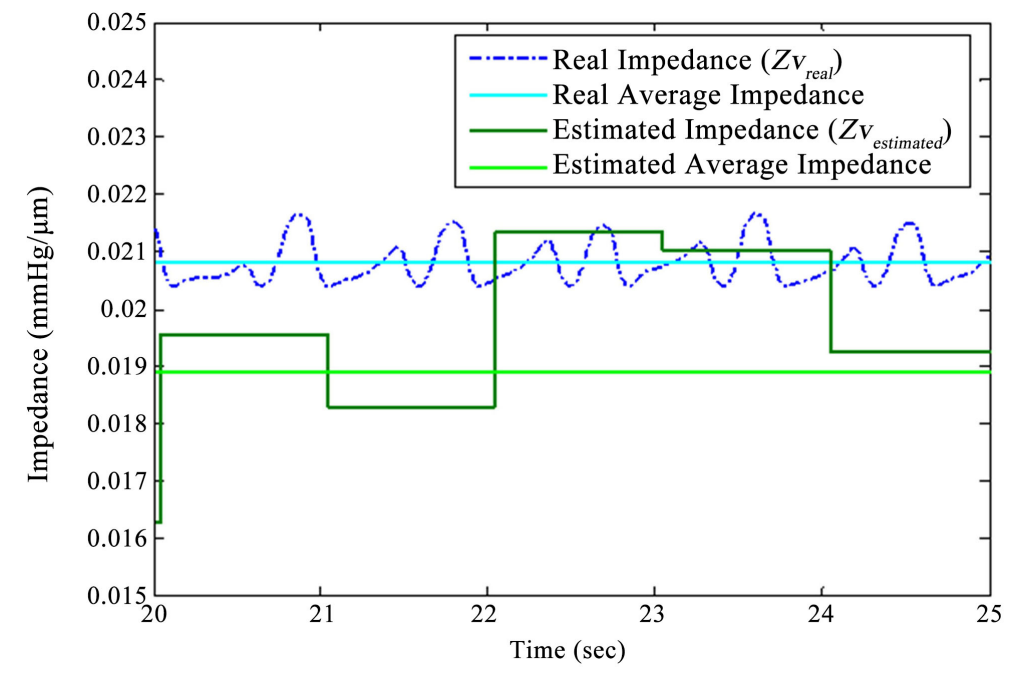

Figure 11. The comparison chart for real and estimated impedance of blood vessel by using TCM.

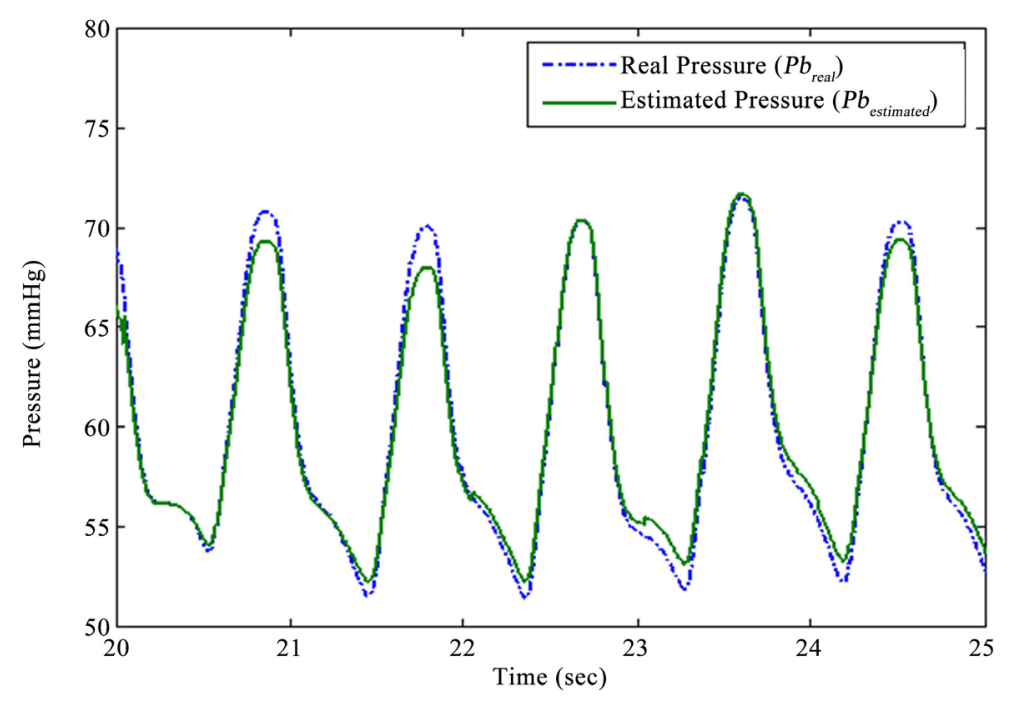

Figure 12. The comparison chart for real and estimated intra-arterial blood pressure by using TCM.

Table 1. Superiority of VLDT over TCM.

\begin{tabular}{|c|c|c|}
\hline Measurements & TCM & VLDT \\
\hline \multirow[t]{2}{*}{ Intra-arterial blood pressure } & $\begin{array}{l}\text { 1. Beat based measurement: } \\
\text { based upon the previous beat of } \\
\text { blood pressure to calculate the } \\
\text { current blood pressure. }\end{array}$ & $\begin{array}{l}\text { 1. Real time based measurement: } \\
\text { using Step-Hold algorithm and featured spline curve fitting technology to identify } \\
\text { the impedance of blood vessel and estimate the blood pressure }\end{array}$ \\
\hline & $\begin{array}{l}\text { 2. Less accuracy: } \\
\text { The estimated error about } \\
\pm 2 \mathrm{mmHg} \text {. }\end{array}$ & $\begin{array}{l}\text { 2. More accuracy: } \\
\text { The estimated error within } \pm 1 \text { mmHg. }\end{array}$ \\
\hline $\begin{array}{l}\text { Dynamic } \\
\text { compliance of blood vessel }\end{array}$ & $\begin{array}{l}\text { 1. Only the average of blood } \\
\text { vessel's impedance is measured. } \\
\text { 2. Can't calculate dynamic } \\
\text { compliance of blood vessel. }\end{array}$ & $\begin{array}{l}\text { 1. The dynamic compliance of blood vessel }\left(C_{3}\right) \text { is measured. } \\
\text { 2. } C_{3} \text { is directly computed by blood vessel's displacement and pressure under } \\
\text { decoupling condition without the effect of surrounding tissues. } \\
\text { 3. it more valuable than } C_{1} \text { and } C_{2} \text { to diagnosing the severity of vascular sclerosis }\end{array}$ \\
\hline
\end{tabular}




\section{Conclusion}

This paper primarily extended the beat-based TCM into the real-time-based VLDT for noninvasively measuring the dynamic impedance of blood vessel and the intra-arterial blood pressure. The advantages of VLDT are decoupling the pulsation of blood vessel from surrounding tissues and using Step-Hold control rules and featured cubic spline curve fitting technique to make real-time measurement feasible. The simulation results show that both estimated errors of impedance of blood vessel and intra-arterial continuous blood pressure are less than $1 \%$; and the extracted dynamic compliance $\mathrm{C} 3$ is more reliable in diagnosing the severity of vascular sclerosis. This could create a promising instrument for clinical researches of circulatory medicine. We require more efforts for constructing the instrument based upon VLDT, and further clinical experiments are planned.

\section{Acknowledgements}

The authors would like to express deep gratitude to Gong Bu Design Company, Ltd. for financial and technical support. In consequence, Gong Bu Design Company, Ltd. reserves all rights to the patent of the dynamic compliance of blood vessel and intra-arterial blood pressure measuring system.

\section{Conflicts of Interest}

The authors declare no conflicts of interest regarding the publication of this paper.

\section{References}

[1] Marey, E.J. (1876) Pressionetvitesse du sang Physiologigue Experimentale Vol. 2. Masson, Paris, 307-343.

[2] Boehmer, R.D. (1987) Continuous, Real-Time, Noninvasive Monitor of Blood Pressure, Peňáz Methodology Applied to the Finger. Journal of Clinical Monitoring, 3, 282-287.

[3] Peňáz, J. (1973) Photoelectric Measurement of Blood Pressure, Volume and Flow in the Finger. Digest of the 10 th International Conference on Medical and Biological Engineering, Germany, Dresden, 104.

[4] Wesseling, K.H. (1984) Non-Invasive Continuous Blood Pressure Wave Form; Measurement by the Method of Penaz. Scr. Medica, 57, 321-334.

[5] Wesseling, K.H. and Peňáz, J. (1986) Indirect Blood Pressure Measurement in the Finger; Physiological Background of a Recent Development. Scr. Medica, 59, 203-206.

[6] Geddes, L.A. (1970) The Direct and Indirect Measurement of Blood Pressure. Year Book Medical Publishers, Chicago.

[7] Shirer, H.W. (1962) Blood Pressure Measuring Methods. IRE Transactions on Bio-Medical Electronics, 9, 116-125. https://doi.org/10.1109/TBMEL.1962.4322975

[8] O’Brien, E. and O’Malley, K. (1991) Handbook of Hypertension: Blood Pressure Measurement. In: Birkenhager, W.H. and Reid, J.L., Eds., Vol. 14, Elsevier, Amsterdam.

[9] Yamakoshi, K.I., Shimazu, H. and Togawa, T. (1979) Indirect Measurement of In- 
stantaneous Arterial Blood Pressure in the Rat. American Journal of Physiology-Heart and Circulatory Physiolog, 237, H632-H637. https://doi.org/10.1152/ajpheart.1979.237.5.H632

[10] Yamakoshi, K.I., Shimazu, H. and Togawa, T. (1980) Indirect Measurement of Instantaneous Arterial Blood Pressure in the Human Finger by the Vascular Unloading Technique. IEEE Transactions on Biomedical Engineering, BME-27, 150-155. https://doi.org/10.1109/TBME.1980.326616

[11] Tanaka, S., Gao, S., Nogawa, M. and Yamakoshi, K.I. (2005) Noninvasive Measurement of Instantaneous Radial Artery Blood Pressure: An Instrument Based on the Volume-Compensation Method. IEEE Engineering in Medicine and Biology Magazine, 24, 32-37. https://doi.org/10.1109/MEMB.2005.1463393

[12] Tanaka, S., Nogawa, M., Yamakoshi, T. and Yamakoshi, K.I. (2007) Accuracy Assessment of a Noninvasive Device for Monitoring Beat-by-Beat Blood Pressure in the Radial Artery Using the Volume-Compensation Method. IEEE Transactions on Biomedical Engineering, 54, 1892-1895. https://doi.org/10.1109/TBME.2007.894833

[13] Gao, S.M., Song, Y.L., Tanaka, S. and Yamakoshi, K.I. (2009) An Instantaneous Blood Pressure Measuring System at the Wrist Based on the Volume-Compensation Method. Chinese Journal of Medical Instrumentation, 33, 323-327.

[14] Lin, A.C.Y., Huang, H.N., Su, Y.C., Shiu, C.Y., Wang, Y.H., Chiang, H.C., Tung, H. and Lin, J.S. (2007) On Measuring the Instantaneous Blood Pressure in an Artery via the Tissue Control Method. Physiological Measurement, 28, 937-951. https://doi.org/10.1088/0967-3334/28/8/015

[15] Cohn, J.N., Finkelstein, S., McVeigh, G., Morgan, D., LeMay, L., Robinson, J. and Mock, J. (1995) Noninvasive Pulse Wave Analysis for the Early Detection of Vascular Disease. Hypertension, 26, 503-508. https://doi.org/10.1161/01.HYP.26.3.503 